Nase
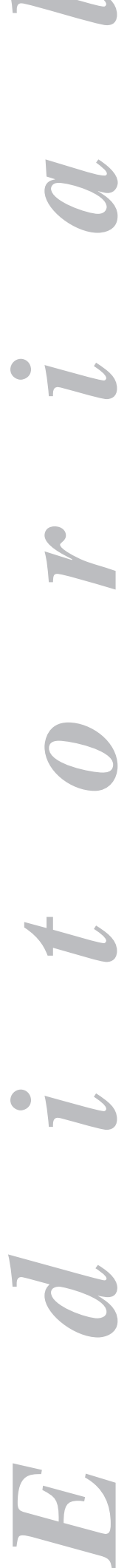

\title{
Should we prevent colorectal cancer?
}

Cancer and cardiovascular disease remain the primary causes of death in western countries.

Colorectal carcinoma (CRC) is among the most prevalent neoplasms in western countries, and 11 million cancers newly develop yearly worldwide, which result in seven million deaths. Predictions for 2030 foresee an incidence twice as high. CRC is the third most common type of cancer with nearly a million new cases per year, and the fourth leading cause of death.

In our country it is the second leading cancer after lung cancer in males and breast cancer in females. Incidence in Spain is estimated in some 25,000 diagnosed cases per year with mortality around $50 \%$, which means that approximately 12,500 patients die annually from CRC in our country; it therefore represents the second leading cause of cancer-related death nationwide (1).

Most CRC cases (70-75\%) are sporadic forms whose triggering factors are still unknown; the remaining 25-30\% of patients may have a family history favorable to the development of this neoplasm. Thus in 3-5\% of cases CRC develops in the setting of a disease with known hereditary background, primarily familial adenomatous polyposis (FAP), with a prevalence of $1 \%$, or as hereditary non-polyposis CRC (HNPCC) or Lynch syndrome, with a prevalence around $2.5 \%$ in Spain. Less than $1 \%$ CRCs develops in patients with inflammatory bowel disease.

Marked regional differences exist, and areas with a high socioeconomic status are most affected -northern Europe, USA and Australia. In these countries the incidence of CRC is four times higher than in developing countries, which ranges from over 40 cases per 100,000 population in Europe to fewer than 5 cases per 100,000 population in Africa or Central America. These incidence rates seem consistent with a country's urban drift- it is estimated that most CRC cases will be diagnosed in economically developed regions by 2030. Observational studies have shown that the incidence of CRC among immigrants from low-risk countries to high-risk countries matches the letters' incidence within one or two generations. Environmental factors play thus a clear key role in the development of CRC.

Therefore, temporal studies and studies in migratory populations suggest that CRC greatly depends on environmental factors, as shown by the highly variable frequencies seen among different countries, and the marked increases in case numbers seen in populations migrating from low-incidence to high-incidence areas. Based on these epidemiological studies, it has been estimated that up to 70-80\% of CRCs may be attributed to the actions of dietary, environmental, and lifestyle factors. This suggests the relevance of potentially modifiable causes that could be prevented to a great extent (2-5).

Diet is one of the most important lifestyle factors, and has been thoroughly studied. 
In 1997 a comprehensive review was published (6), and new relevant findings have subsequently been reported.

Thus, clinical trials with different dietary interventions (e.g., increased fiber, fruits, and vegetables, decreased fat, vitamin and/or mineral supplementation) have shown inconclusive results. However, several research lines support the role diet modifications may play in the prevention of this type of tumor. For instance, many observational studies suggest that vegetables, fruits, a fiber-rich diet, and certain micronutrients may protect against CRC, whereas alcohol and red meat (and the way it is cooked) may increase its risk. Similarly, several animal studies have confirmed that some dietary interventions may reduce cancer risk (7-10).

- Fruits, vegetables, and fiber. While until relatively recently case-control studies had seemed to suggest that fruits and vegetables might decrease the risk for CRC, cohort studies have shown conflicting results (some benefit in some, no benefit in others). Overall, the use of these foods may perhaps play a small preventive role for the development of CRC.

Fiber ingestion has been linked to a reduced risk for CRC based on epidemiological evidences (lower incidence of CRC in underdeveloped societies where fiber ingestion is high) and pathogenetic theories (faster intestinal transit would preclude the contact of carcinogens with the colonic mucosa). Case-control studies showed a protective role of fiber, whereas a recent pooled analysis of published cohort studies in over 8,000 cases could not demonstrate such beneficial effect. An explanation for these diverging results is maybe that despite an identical amount of fiber in the analyzed cohorts, fiber composition might differ widely (soluble or non-soluble, from cereals, fruits or vegetables). On the other hand, fiber supplementation has not proven effective in the prevention of colon adenoma recurrence (11-13).

- Association with red meat and fat ingestion. The link between red meat (lamb, beef, pork) and CRC shows conflicting results, probably because of the inclusion of processed and manufactured meat (e.g., sausage) in some studies, and varying meat preparation techniques. Recent studies with extensive cohorts do show a weak association between red meat consumption and CRC.

It is believed that the different methods of cooking red meat (fried, grilled) generate different mutagenic substances (heterocyclic amines, polycyclic aromatic hydrocarbons) within meat; alternatively, people with a rapid acetylator genotype might be more susceptible to meat carcinogens.

There seems to be a clear association between processed meat consumption and risk of CRC, quantified as a $50 \%$ increase for a daily ingestion of $25 \mathrm{~g}$. It is speculated that this risk may be due to the presence of nitrosamines in cured meat $(14,15)$.

Fat ingestion could not be linked to increased CRC risk.

- Dairy products and calcium. Consumption of dairy products has been associated with a $11 \%$ decrease in CRC risk by a pooled analysis of 10 cohort studies including 5,000 cases. This association has also been seen with calcium supplements, which reduced colon adenoma recurrence by $19 \%$ versus placebo (16).

On the other hand, excessive alcohol consumption, particularly when associated with a diet poor in folic acid and methionine, and exposure to tobacco smoke, also increases the risk for CRC. Lack of physical activity and overweight may also represent risk factors for CRC.

Overall, the role of environmental factors in the etiology of CRC seems clearcut. If we could only identify and modify such factors, then we might be able to prevent a huge number of CRCs. 
To sum it all up, studies suggest that the primary prevention of colon cancer is feasible at least to a certain extent, and it is estimated that up to $70 \%$ of cases could be prevented with moderate changes in diet and lifestyle. Therefore, currently available data warrant interim recommendations regarding diet and lifestyle, which combined with early diagnosis methods, preventive therapy, and follow-up, might obtain excellent results against this common, complex disease that is also preventable to a great extent.

Furthermore, customized, more effective recommendations will be possible in a near future as our understanding increases regarding the association between specific genetic patterns and a higher or lower predisposition to the causal or protective actions of the various environmental factors involved.

Therefore, considering current data, we should recommend primary prevention with diet and lifestyle modifications for CRC:

- Red meat (particularly processed meat: cured, smoked, salted, canned meat) consumption, perhaps depending on how it is cooked, is associated with an increased risk for CRC via unknown mechanisms.

- While data from reported studies are inconclusive, a fiber-rich diet may decrease the risk for CRC in addition to other beneficial effects at both the gastrointestinal and systemic levels.

- Data are also inconclusive regarding fruits and, most particularly, vegetables, but these foods might protect from CRC development.

- Calcium supplementation in the presence of adequate vitamin D levels may help in the prevention of CRC.

- The presence of adequate folic acid and methionine levels may protect from CRC development. Folic acid supplementation, particularly in some groups (alcohol abuse, some patients with inflammatory bowel disease), may help reach this goal.

- Except for folic acid and calcium supplementation, data are insufficient to recommend for or against other supplements (e.g., antioxidant vitamins) with the aim of decreasing CRC development.

- Smoking increases the risk of CRC.

- Alcohol consumption, particularly when excessive and in combination with reduced folic acid levels, increases the risk of CRC.

- Obesity increases the risk of CRC whereas physical activity does the opposite. Physical exercise is advisable, as is avoiding being overweight, to prevent the development of CRC (17-19).

In the present issue of the Spanish Journal of Gastroenterology, Béjar et al. report on the results of a worldwide dietary analysis (20 countries) assessing the incidence of CRC and then the annual per-capita ingestion of pure alcohol (in liters), cereal, fruits, vegetables, pulses, poultry, red meat, and fish to estimate the risk for developing CRC (20).

Middle-aged males have an estimated risk of CRC of $71 \%$ attributable to the following six factors -red meat ingestion, low folic acid intake, alcohol consumption, obesity, physical inactivity, and early-onset smoking.

Epidemiological evidence has identified a number of both risk and protective dietary factors for colorectal cancer, as well as a huge potential for primary prevention based on changes in the exposure to such factors (3-8). The evolution of consumption for the various dietary variables studied is potentially consistent with the incidence rate trends seen in analyzed countries, which are then framed in one of the four previously mentioned situations.

The great challenge entailed in performing this type of epidemiological studies based on population surveys for risk groups must be highlighted, particularly given 
the wide scope of the questions posed. This renders extremely difficult the establishment of causality correlations given the relevant bias involved in answering with rigor and reliability the questions included.

Notwithstanding, this does not detract a bit from the merit, interest, and quality of the reported study, or the effort invested in its design and conduction, as well as in interpreting the results obtained. Fair enough, this is the first study in Spain on this interesting subject, and it should stand as an example and stimulus for further research in the various regions of our country.

Two excellent studies on this matter were also published 2 and 4 years ago, where potentially involved dietary factors were discussed in detail $(18,19)$.

While many mechanisms remain to be elucidated, there is some evidence from epidemiological and experimental studies that a number of dietary, environmental, and lifestyle factors play a relevant role in the development of CRC.

We are confident that this report will serve as a stimulus for further research by new teams at a national and international level in an attempt to find meaningful correlations between diet and CRC (let us not forget that $70 \%$ of cases are sporadic), and even to demonstrate the potential benefits of the Mediterranean diet for its prevention.

Therefore, CRC has shown a high prevalence and mortality that are further increasing, and countries are logically implementing measures to either prevent its development or detect it as early as possible; this is why extra efforts should be invested in this type of studies to investigate causal factors, and in techniques for an early diagnosis of precursor lesions.

Pedro J. Tárraga-López

Associated Professor of Medicine, Universidad de Castilla la Mancha. Family Practitioner. Albacete, Spain

\section{REFERENCES}

1. Internacional Agency for Research on Cancer (IARC), 2010. Available at: http://www-dep.iarc.fr/

2. Parkin DM. International variation. Oncogene 2004; 23: 6329-40.

3. Parkin DM, Olsen AH, Sasieni P. The potential for prevention of colorectal cancer in the UK. Eur J Cancer Prev 2009; 18:179-90.

4. Lynch HT, De la Chapelle A. Hereditary colorectal cancer. N Engl J Med 2003; 348: 919-32.

5. Anwar S, White J, Hall C, Farrell WE, Deakin M, Elder JB. Sporadic colorectal polyps: management options and guidelines. Scand J Gastroenterol 1999;34:4-11.

6. Martinez ME. Primary prevention of colorectal cancer: lifestyle, nutrition, exercise. Recent Results Cancer Res 2005;166:177-211.

7. Cho E, Smith-Warner SA, Ritz J, Van den Brandt PA, Colditz GA, Folsom A, et al. Alcohol intake and colorectal cancer: a pooled analysis of 8 cohort studies. Ann Intern Med 2004;140:603-13.

8. Van den Brandt PA, Goldbohm RA. Nutrition in the prevention of gastrointestinal cancer. Best Pract Res Clin Gastroenterol 2006;20:589-603.

9. Bergstrom A, Pisani P, Tenet V, Wolk A, Adami HO. Overweight as an avoidable cause of cancer in Europe.Int J Cancer 2001;91:421-30.

10. Gunter MJ, Leitzmann MF. Obesity and colorectal cancer: epidemiology, mechanisms and candidate genes.J Nutr Biochem 2006;17:145-56.

11. McTiernan A. Obesity and cancer: The risks, science, and potential management strategies. Oncology 2005; 19: 871-81.

12. Park Y, Hunter DJ, Spiegelman D, Bergkvist L, Berrino F, Van den Brandt PA, et al. Dietary fiber intake and risk of colorectal cancer: a pooled analysis of prospective cohort studies. JAMA 2005;294:2849-57.

13 Alberts DS, Martínez ME, Roe DJ, Guillén-Rodríguez JM, Marshall JR, Van Leeuwen JB, et al. Lack of effect of a high-fiber cereal supplement on the recurrence of colorectal adenomas. Phoenix Colon Cancer Prevention Physician's Network. N Engl J Med 2000;342:1156-62. 
14. Norat T, Bingham S, Ferrari P, Slimani N, Jenab M, Mazuir M, et al. Meat, fish and colorectal cancer risk: The European Prospective Investigation into Cancer and Nutrition. J Natl Cancer Inst 2005;97:906-16.

15. Sandhu MS, White IR, McPherson K. Systematic review of the prospective cohort studies on meat consumption and colorectal cancer risk: a meta-analytical approach. Cancer Epidemiol Biomarkers Prev 2001;10:439-46.

16. Cho E, Smith-Warner SA, Spiegelman D, Beeson WL, Van den Brandt PA, Colditz GA, et al. Dairy foods,calcium and colorectal cancer: A pooled analysis of 10 cohort studies. J Natl Cancer Inst 2004;96:1015-22.

17. Solera Albero J, Tárraga López PJ, López Cara MA, Celada Rodríguez A, Cerdán Oliver M, Ocaña López JM. Influencia de la dieta y los estilos de vida en el cáncer colorrectal. Rev Esp Enferm Dig 2007;99:190-200.

18. Maté Jiménez J, Rodríguez Muñoz S, Mearin F, Pajares JM. Frecuencia del carcinoma de colon y de estómago en el período 1960-1979 en relación con los cambios dieteticos y migratorios. Rev Esp Enferm Dig 1987;71:31-7.

19. Franco A, Sikalidis AK, Solís Herruzo JA. Colorectal cancer: influence of diet and lifestyle factors. Rev Esp Enferm Dig 2005;97:432-48.

20. Bejar LM, Gili M, Infantes B, Marcott PF. Effects of changes in dietary habits on colorectal cancer incidence in twenty countries from four continents during the period 1971-2002. Rev Esp Enferm Dig 2011;103(10): 519-29. 